\title{
Explosive crystallization mechanism of ultradisperse amorphous films
}

\author{
A.I. Olemskoi ${ }^{\mathrm{a}, *}$, A.V. Khomenko ${ }^{\mathrm{a}}$, V.P. Koverda ${ }^{\mathrm{b}}$ \\ ${ }^{a}$ Physical Electronics Department, Sumy State University, Rimskii-Korsakov St., 2, \\ 244007 Sumy, Ukraine \\ ${ }^{\mathrm{b}}$ Institute of Thermal Physics, Urals Branch of Russian Academy of Sciences, \\ 620219 Ekaterinburg, Russia
}

Received 22 December 1999

\begin{abstract}
The explosive crystallization of germanium ultradisperse amorphous films has been studied experimentally. We show that crystallization may be initiated by local heating at the small film thickness but is realized spontaneously at large thickness. The fractal pattern of the crystallization phase is discovered to be inherent in the phenomena of diffusion-limited aggregation. It is shown that in contrast to the ordinary crystallization mode, the explosive one is connected with the instability which is caused by self-heating. A transition from the first mechanism to the second one is modelled by the Lorenz system. The process of explosive crystallization is represented on the basis of the self-organized criticality conception. The front movement is described as the effective diffusion in the ultrametric space of hierarchically subordinated avalanches, corresponding to the explosive crystallization of elementary volumes of ultradisperse powder. The expressions for the stationary crystallization heat distribution and the steady-state heat current are obtained. The heat needed for initiation of the explosive crystallization is obtained as a function of the thermometric conductivity. The time dependence of the spontaneous crystallization probability in a thin film is examined. (c) 2000 Elsevier Science B.V. All rights reserved.
\end{abstract}

PACS: 81.30.-t; 05.40.+j; 64.60.Lx

Keywords: Explosive crystallization; Self-organized criticality; Ultrametric space

\section{Introduction}

The metastable amorphous films are obtained usually by the quenching of a melt or by steam condensation on a cold substrate [1-4]. Experiments show a vast variety

\footnotetext{
* Corresponding author.

E-mail addresses: olemskoi@ssu.sumy.ua (A.I. Olemskoi), iva@itp.e-burg.su (V.P. Koverda)
} 
and complicated character of the subsequent transitions into the stable crystalline state [1-6]. If the film thickness is so small that the crystallization heat can be absorbed by a thermostat, the ordinary cold crystallization mechanism works [1]. So, in the crystallized films of semiconductors the undulating surface is developed under the formation of combs, whose long axes are perpendicular to the direction of the crystallization front movement [5]. As is known [5,6], at small number of the crystal embryos this mechanism is realized if the nucleation frequency of crystallization centers $J$ is very small.

Sometimes, explosive crystallization can be initiated by local heating (for example, by laser or electron impulse). Such a scenario takes place in the case of instability appearance of the interfacial boundary motion due to both the heat exchange with substrate and the influence of laser radiation [5,6]. This instability is ensured with a nonlinear dependence of the crystal growth velocity $u$ as a function of temperature. Moreover, the crystallization front instability can be fluctuating in character that manifests itself in the experiments with undulation of crystallized surface $[5,6]$. Such a behaviour appears in the partially crystallized film or at the incomplete crystallization of amorphous phase.

Another scenario is observed in the amorphous medium in which as the crystal growth velocity $u$, the nucleation rate $J$ of embryonic crystals is sufficiently large. Indeed, at low temperatures, the quantities $J$ and $u$ increase with temperature growth, so that self-heating stimulates crystallization. Therefore, the increase of a film thickness can lead to a situation, when the crystallization heat cannot be absorbed by an environment which causes the heat instability [7]. As a result, the spontaneous transition to a regime of the explosive crystallization can be due to the heating effect. Examples of such amorphous mediums are the amorphous ice layers, some organic matter $[1,3]$, and the layers of the germanium amorphous ultradisperse powder with the admixture of the crystalline phase $[3,4]$.

Our work is devoted to studying the explosive crystallization mechanism produced by such type of instability.

The experimental data in Section 2 show that the course of explosive crystallization of ultradisperse amorphous materials is determined by the high density of crystalline phase embryos. The crystallization phase spreading is similar to percolation cluster formation using the liquid flow in a random medium [8]. The formed cluster has the branching fractal structure that is characteristic for the thermal conductivity limited aggregation. Our approach is based on the assumption that such structure leads to the hierarchical picture of the explosive crystallization process.

In Section 3, investigation of the conditions required for this scenario, as a result of self-organization, is carried out. This process is fixed by the velocity of crystallization front motion, its temperature, and differences in the thermodynamic potentials of amorphous and crystalline states. The first of the mentioned parameters is connected with the third one by means of the positive feedback which is the reason for selforganization. The connection between the first and second parameters is due to the negative feedback reflecting the Le Chatelier principle. As a result of the interplay between the factors pointed out, the stationary state is established at supercritical 
value of transformation thermal energy, where the crystallization front velocity can take anomalous large values. Then, at small film thickness, a regime is realized when the crystallization process can be initiated only by the external influence type of the laser beam [2]. However, with film growth up to the critical value, the crystallization heat in the film volume becomes sufficient for a spontaneous increase in the front velocity. Such a situation is observed experimentally in Ref. [3].

In Section 4 the direct examination of explosive crystallization is carried out as a self-organized criticality process caused by stochastic heat spreading over hierarchical tree nodes. The study of effective motion equation shows that, in accordance with the result of the previous section, instability develops in the case, when crystallization heat effect (or externally inputted energy) is above a critical quantity determined by the thermometric conductivity. The stationary crystallization heat distribution, defined as a solution of corresponding Fokker-Planck equation, allows us to find both the heat current arising during crystallization and the probability of spontaneous crystallization in a film with subcritical thickness. According to Section 5, the probability increases logarithmically as a function of time until the maximum value. On its turn, this value decreases monotonically with the growth of thermometric conductivity.

\section{Experimental results}

The experimental study of influence of the crystal inclusions distributed in the amorphous phase volume in the kinetics of the explosive crystallization has been carried out with germanium [4]. Unfortunately, there are no information in literature about spontaneous rise of the explosive crystallization in continuous amorphous thin films of germanium. It is known that the maximum value of formation frequency of crystalline embryos is only $J_{\max } \sim 10^{14} \mathrm{~s}^{-1} \mathrm{~cm}^{-3}$ in the supercooled germanium and corresponds to the higher temperatures than realized at the explosive crystallization process [1]. Therefore, in the germanium amorphous films the natural process of crystals nucleation has not enough intensity for a significant influence in the explosive crystal growth. Let us point out in this connection that in amorphous ice layers, where the spontaneous explosive crystallization takes place, one has $J_{\max } \sim 10^{20} \mathrm{~s}^{-1} \mathrm{~cm}^{-3}$.

In order to intensify the mentioned influence of crystal inclusions, the experiments were carried out with thin films of amorphous nanopowders with admixture of large number of smallest crystals having no more than $3-10 \%$ of total mass. The layers of amorphous powder with the characteristic size of particles 3-10 nm were obtained by thermal evaporation and following condensation of germanium in the atmosphere of inert gas at pressures 10-100 Pa. Changing the evaporation intensity allows us to regulate the content of the crystalline particles in amorphous powder. Another peculiarity of our experiments is that the substrates absorb significantly smaller heat due to the porosity of amorphous films.

Spontaneous explosive crystallization has been observed in layers of nanopowders with thickness $0.01-0.1 \mathrm{~mm}$ at the substrate temperature $300-400 \mathrm{~K}$. Depending on 


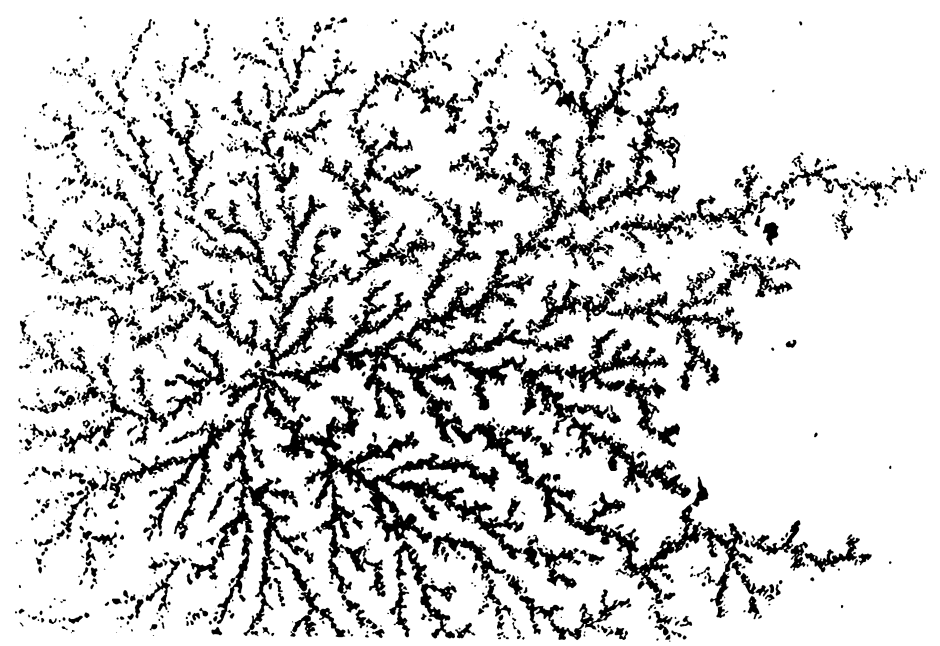

(a)

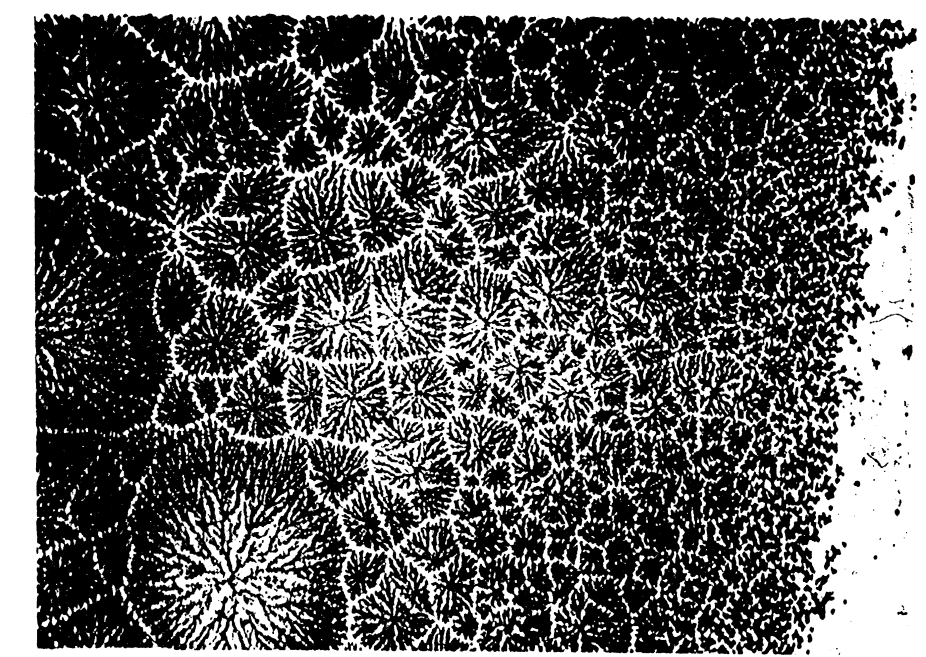

(b)

Fig. 1. The patterns which arise in the layer of amorphous powder of Ge at the explosive crystallization: (a) from single center; and (b) from several centers which arise spontaneously. Magnification $=12.5$.

the initial concentration of crystalline phase, the movement velocities of crystallization wave have been changed in the range $0.01-0.1 \mathrm{~m} \mathrm{~s}^{-1}$. In contrast, the transverse undulation being inherent in the usual crystallization mechanism, in our case the front movement leads almost always to formation of "twigs" along the movement direction. The characteristic features of explosive crystallization in the powder layers are shown in Fig. 1, where the light background corresponds to non-crystallized domains. It is seen that the rise of explosive crystallization avalanches occurs from the single centers 
which act as original embryos. The cornerstone of our observation is that the crystalline phase distribution has a fractal character it being similar to the pattern appeared in diffusion limited aggregation [8].

\section{Determination of explosive crystallization conditions}

The experimental data show that two mechanisms of amorphous material crystallization, depending on external conditions and the presence of crystalline phase embryos, are possible: the slow growth of a cold crystal and the explosive crystallization that is caused by the phase transformation heating. According to Ref. [4], the transition between these regimes is jump-like in character, as a first-order phase transition. We will show below that such a transition is caused by the system self-organization due to the positive feedback between the heating and growth velocity of crystalline phase.

To analyze the problem, let us examine the time dependences of the crystallization front velocity $u(t)$, its temperature $T(t)$, and the specific crystallization heat $f(t)$. The equations defining these dependences take into account their dissipative character and the positive feedback between quantities $u$ and $f$, that is the reason for self-organization. On the other hand, in order to provide stability for a system we introduce also the negative feedback between $u$ and $T$. The equations obtained as a result coincide formally with the Lorenz system that is the simplest way to describe the self-organization process [9].

The first of the stated equations has the form

$$
\dot{u}=-u / \tau_{u}+\mu T,
$$

where the dot stands for a derivative with respect to time $t, \mu>0$ is a constant. The first term in the right-hand side describes the Debye relaxation during time $\tau_{u}$, the second one reflects the increase in the crystallization front velocity with the growth of temperature difference $T=T_{0}-T_{\infty}$ at the crystallization front and the thermostat, respectively. In the stationary state $\dot{u}=0$ and Eq. (3.1) gives linear relationship $u=A_{u} T$, where the constant $A_{u} \equiv \tau_{u} \mu$ is introduced.

The equation for the rate of quantity $T$ variation has the nonlinear form

$$
\dot{T}=-T / \tau_{T}+g_{T} u f,
$$

where $f>0$ is the volume density of difference of the thermodynamic potentials of amorphous and crystalline states; $\tau_{T}, g_{T}$ are positive constants. As in Eq. (3.1), the first term on the right-hand side of Eq. (3.2) describes the relaxation process of temperatures difference $T$ to the stationary value $T=0$. It takes place not during the macroscopic time $\tau_{u}$ but during the mesoscopic one $\tau_{T}$, so that the important requirement for the future condition $\tau_{T} \ll \tau_{u}$ is satisfied. The second term describes the mentioned positive feedback between the crystallization front velocity $u$ and the difference $f$ between the specific thermodynamic potentials of the phases that results in increase of the value $T$ 
and, thus, causes the self-organization process. In the stationary case $\dot{T}=0$, Eq. (3.2) takes the form

$$
T_{0}=T_{\infty}+A_{T} u f
$$

where the constant $A_{T} \equiv g_{T} \tau_{T}$ is introduced. According to Eq. (3.3), the non-linear term describes the heating of the crystallization front with the growth of crystallization wave velocity.

The kinetic equation for the difference $f$ of specific thermodynamic potentials

$$
\dot{f}=\left(f_{0}-f\right) / \tau_{f}-g_{f} u T
$$

differs from Eqs. (3.1) and (3.2) as follows: the relaxation of quantity $f$ occurs not to the zero but to the finite value $f_{0}$, representing the energy density inputted in the system (heat effect of transformation); $\tau_{f}$ is a corresponding relaxation time. In Eq. (3.4) the negative feedback between the quantities $u$ and $T$ is introduced to the decrease of thermodynamic transformation factor $f$ with the growth of the crystallization front velocity and its temperature ( $g_{f}>0$ is a corresponding constant).

Let us study the system of differential equations (3.1), (3.2), (3.4) defining the self-consistent behaviour of the quantities $u(t), T(t), f(t)$ which act as the order parameter, the conjugate field, and the control parameter, respectively [9]. With this aim, we write the Lorenz system in the form

$$
\begin{aligned}
& \tau_{u} \dot{u}=-u+A_{u} T, \\
& \tau_{T} \dot{T}=-T+A_{T} u f, \\
& \tau_{f} \dot{f}=\left(f_{0}-f\right)-A_{f} u T,
\end{aligned}
$$

where the constant $A_{f} \equiv \tau_{f} g_{f}$ is introduced. As is known [9], the analytic examination is possible only if the hierarchical subordination conditions are satisfied

$$
\tau_{T} \ll \tau_{u}, \quad \tau_{f} \ll \tau_{u},
$$

which means that in the course of evolution the temperatures difference $T$ and the thermodynamic potential $f$ follow the variation of the crystallization front velocity. As was mentioned above, the first of these conditions is always obeyed. Since, on the other hand, $\tau_{f} \sim \tau_{T}$, the second inequality (3.6) is met also.

When the values of relaxation times $\tau_{u}, \tau_{T}, \tau_{f}$ are constant and conditions (3.6) are obeyed, it is not difficult to see that the system of equations (3.5) describes the second-order transition. However, the cold crystallization mode transforms into the explosive one in accordance with the first-order mechanism. To avoid this discrepancy, let us use the simplest approximation [10]

$$
\frac{1}{\tau_{u}}=\frac{1}{\tau_{0}}\left(1+\frac{\kappa}{1+\left(u / u_{\tau}\right)^{2}}\right),
$$

characterized by the positive constants $\tau_{0}, \kappa$, and $u_{\tau}$. Moreover, it is convenient to introduce the scales of quantities $u, T$, and $f$ :

$$
u_{m} \equiv\left(A_{T} A_{f}\right)^{-1 / 2}, \quad T_{m} \equiv u_{m} / A_{u}=A_{u}^{-1}\left(A_{T} A_{f}\right)^{-1 / 2}, \quad f_{c} \equiv\left(A_{u} A_{T}\right)^{-1}
$$


where $A_{u} \equiv \tau_{0} \mu$. Then, system (3.5) assumes the simplest form

$$
\begin{aligned}
& \tau_{0} \dot{u}=-u\left[1+\kappa\left(1+u^{2} / \alpha^{2}\right)^{-1}\right]+T, \\
& \tau_{T} \dot{T}=-T+u f, \\
& \tau_{f} \dot{f}=\left(f_{0}-f\right)-u T,
\end{aligned}
$$

where the parameter $\alpha \equiv u_{\tau} / u_{m}$ is introduced.

Taking into account conditions (3.6), the left-hand sides of Eqs. (3.10) and (3.11) can be set equal to zero because of the small relaxation times $\tau_{T}, \tau_{f}$. As a result, we obtain the expressions for the temperature difference $T$ and the thermodynamic potential $f$ in terms of velocity $u$ of the crystallization front

$$
\begin{aligned}
& T=f_{0} u\left(1+u^{2}\right)^{-1}, \\
& f=f_{0}\left(1+u^{2}\right)^{-1} .
\end{aligned}
$$

At $u \ll 1$, dependence (3.12) has the linear form characterized by the susceptibility $\partial u / \partial T=f_{0}^{-1}$. At $u=1$, function $T(u)$ becomes saturated, and at $u>1$ decreasing dependence is realized that has no physical meaning. This implies that the constant $u_{m}$ defined in (3.8) has the physical meaning of the maximum value of the crystallization front velocity. According to Eq. (3.13) the difference $f$ of specific thermodynamic potentials of different phases decreases monotonically with the growth of velocity $u$ from the value $f_{0}$ at $u=0$ to $f_{0} / 2$ at $u=1$. Obviously, this decrease is caused by the negative feedback in Eq. (3.11) which is the reflection of Le Chatelier principle for the examined problem. On the other hand, the positive feedback between the velocity $u$ and the thermodynamic factor $f$ in Eq. (3.10) is the reason for transition to the explosive crystallization mechanism that leads to the growth of $T$ due to the crystallization front heating. However, in accordance with Eq. (3.11), the latter results in decrease of $f$ as a consequence of the self-organization process.

Within the framework of the adiabatic approximation $\tau_{T}, \tau_{f} \ll \tau_{0}$, the Lorenz system (3.9) $-(3.11)$ is reduced to the Landau-Khalatnikov equation

$$
\tau_{0} \dot{u}=-\partial V / \partial u \text {. }
$$

Its form is determined by the effective potential

$$
V=\frac{1}{2}\left[u^{2}-\theta \ln \left(1+u^{2}\right)\right]+\frac{\kappa \alpha^{2}}{2} \ln \left[1+(u / \alpha)^{2}\right],
$$

where $\theta \equiv f_{0} / \Delta h, \Delta h \equiv\left(\tau_{0} \tau_{T} g_{u} g_{T}\right)^{-1}$ is the scale defining the specific crystallization heat, the quantity $V$ is measured in units of $u_{m}^{2}$. For small values of $\theta$ the curve of the $V$ vs. $u$ dependence has a monotonically increasing shape with its minimum at point $u=0$ that corresponds to the cold crystallization mechanism. At $\theta=\theta_{c}^{0}$, where

$$
\theta_{c}^{0} \equiv 1+\alpha^{2}(\kappa-1)+2 \alpha \sqrt{\kappa\left(1-\alpha^{2}\right)}
$$

a plateau appears, which for $\theta>\theta_{c}^{0}$ is transformed into a minimum corresponding to the velocity $u_{e} \neq 0$ and a maximum at point $u^{m}$ separating a minima which meets the 
values $u=0$ and $u=u_{e}$. When the parameter $\theta$ increases still further, the minimum at point $u=u_{e}$ is lowered and the height of barrier at $u=u^{m}$ decreases, vanishing at the critical value

$$
\theta_{c}=1+\kappa .
$$

The steady-state values of the crystallization front velocity have the form

$$
\begin{aligned}
& u_{e}^{m}=u_{00}\left\{1 \mp\left[1+\left(\frac{\alpha}{u_{00}^{2}}\right)^{2}\left(\theta-\theta_{c}\right)\right]^{1 / 2}\right\}^{1 / 2}, \\
& u_{00}^{2} \equiv \frac{1}{2}\left[(\theta-1)-(1+\kappa) \alpha^{2}\right] .
\end{aligned}
$$

As is shown in Fig. 2a, if the system's energy increases slowly, the jump from zero to $\sqrt{2} u_{00}$ is observed at point $\theta=\theta_{c}$ and then the value $u_{e}$ increases smoothly. If the parameter $\theta$ goes downward quasistatically, the crystallization front velocity $u_{e}$ smoothly decreases up to the point, where $\theta=\theta_{c}^{0}$ and $u_{e}=u_{00}$, and then jumps down to zero. The hysteresis of such type takes place only at the presence of energy barrier inherent in a first-order transition and appears if only the parameter $\alpha=u_{\tau} / u_{m}$ is smaller than unity.

The key point of the studied transition is that the stationary value of the thermodynamic transition factor

$$
f_{e}=\frac{\left(1+u_{00}^{2}\right)-\sqrt{\left(1+u_{00}^{2}\right)^{2}-\theta\left(1-\alpha^{2}\right)}}{1-\alpha^{2}}
$$

equals the thermal energy $\theta$ in the $0<\theta<\theta_{c}^{0}$ interval (Fig. 2b). At $\theta>\theta_{c}^{0}$ this quantity decreases smoothly from the value

$$
f_{m}=1+\alpha\left[\kappa /\left(1-\alpha^{2}\right)\right]^{1 / 2}
$$

at $\theta=\theta_{c}^{0}$ to 1 at $\theta \rightarrow \infty$.

Under quasistatic growth of parameter $\theta$ from 0 to $\theta_{c}$ the stationary value of transformation factor increases linearly being in the same interval. After jump-down at $\theta=\theta_{c}$ the quantity $f_{e}$ decreases smoothly according to dependence (3.19). Under reverse decrease of $\theta$ the quantity $f_{e}$ undergoes the jump at point $\theta_{c}^{0}$ from the value $f_{m}$ up to the $\theta_{c}^{0}$. Since in the important range of values of the parameters $\alpha$ and $\kappa$ limited by $\kappa_{\min }=\alpha^{2} /\left(1-\alpha^{2}\right)$, the maximum value $f_{m}$ of specific transition energy is smaller than the minimum value $\theta_{c}^{0}$ of the heat density, the stationary value $f_{e}$ of the specific difference of thermodynamic potentials of amorphous and crystalline states is always smaller than heat density $\theta$.

The above analysis shows that the effective potential $V(u)$ has the barrier separating the cold and the explosive crystallization modes. As heat density $\theta$ becomes greater than the critical value $\theta_{c}$ this barrier disappears. Thus, at $\theta<\theta_{c}$ the transition to the explosive crystallization mechanism requires the penetration of energy barrier and in the opposite case it is realized spontaneously. The first of the appointed situations takes place in the case when the explosive crystallization is initiated by an external beam 

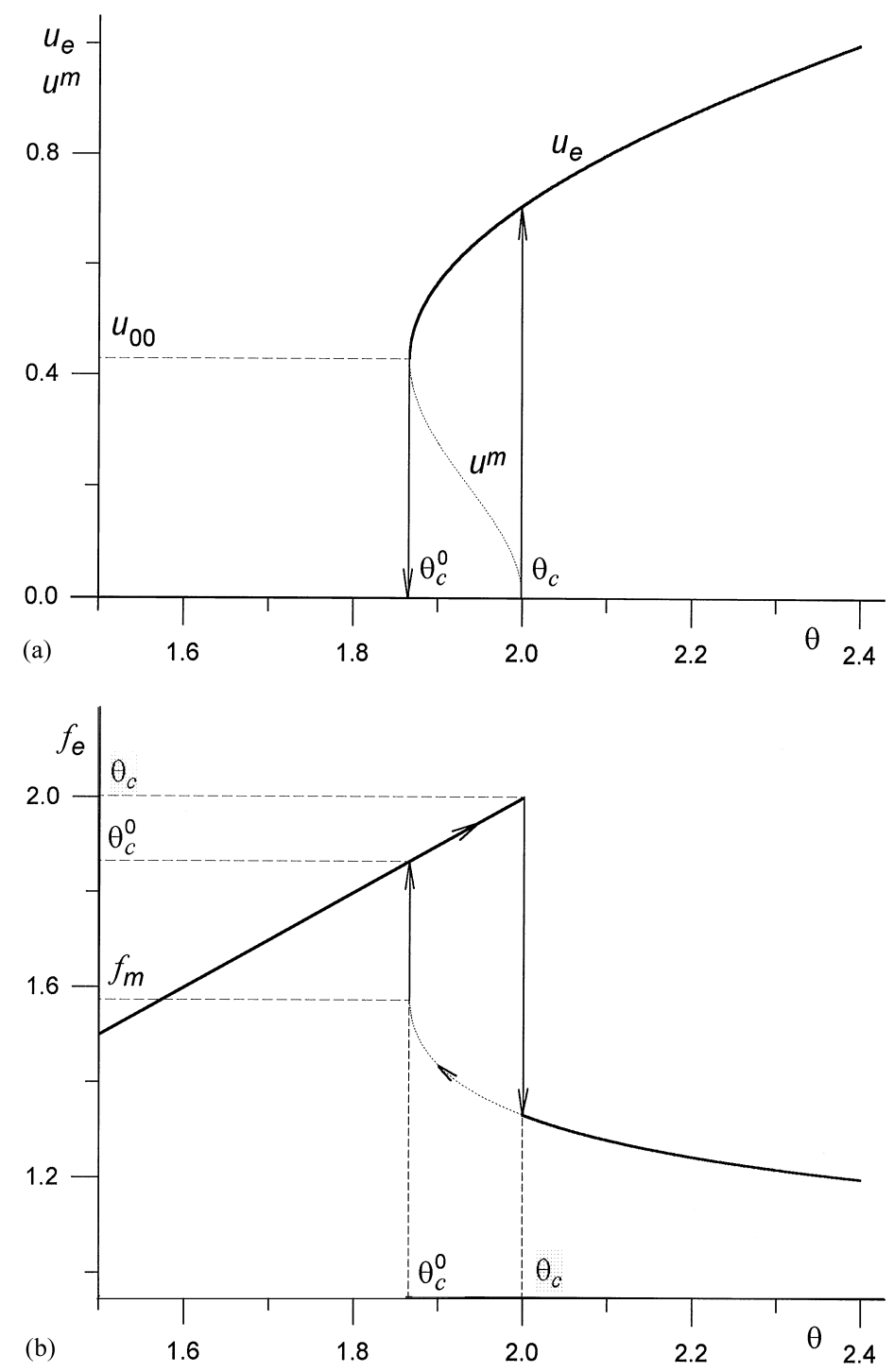

Fig. 2. (a) The dependence of the steady-state velocities of crystallization front on the transition heat (the solid curve corresponds to the stable state $u_{e}$, the dotted curve meets the unstable one $u^{m}$ ); and (b) the dependence of the stationary difference $f_{e}$ of specific thermodynamic phase potentials on the transition heat. The arrows indicate the hysteresis loop.

(see Fig. 1a). With the increase of the coating thickness the crystallization heat cannot be absorbed by substrate and parameter $\theta$ increases. This leads to the scenario that the value $\theta_{c}$ (at which the function $V(u)$ loses barrier) is reached at the critical film thickness and the system transforms into the explosive crystallization regime spontaneously (Fig. 1b). 


\section{Description of explosive crystallization as a self-organized criticality process}

In recent years considerable study has been given to the conception of self-organized criticality (SOC) representing the natural development of critical phenomena picture $[11,12]$. The basic distinction of the SOC from the phase transition is that SOC process is realized spontaneously, whereas the phase transition goes on under the external influence (for instance, the temperature variation). In this section, we will expound the quantitative picture within the framework of which explosive crystallization will be represented as SOC. The basis for such representation is that the SOC process consists of the hierarchically subordinated sequence of elementary phase transitions which are usually called avalanches [13]. Their hierarchical subordination manifests itself in that the avalanches of upper level are formed after their formation has been finished on the lower one. Then this process recurs on the more upper levels - right up to the global avalanche formation on the top of hierarchical tree. The hierarchical nature of explosive crystallization process is discovered obviously in the microscopic photographs of crystallization pattern in Fig. 1, where it appears as the tree-like fractal structure. In accordance with Ref. [14] the hierarchical tree represents the geometrical shape of ultrametric space in that system's states are realized. Thus, for a description of explosive crystallization the geometrical picture of the nodes distribution over hierarchical tree levels is necessary to represent, at first Ref. [15].

Let the maximum number of nodes $N$ be on the bottom hierarchical level corresponding to the distance in the ultrametric space $s=0$. This level meets the elementary avalanches whose number coincides with $N$. This is the only node on the top level ( $s=s_{0} \gg 1$ ) corresponding to the global avalanche. The problem is to find the dependence $N(s)$ that defines the distribution of tree node number over the hierarchical levels.

At first, we examine the basic types of the trees (Fig. 3): regular tree with integer branching ratio $j$, regular Fibbonachi tree with fractional $j=\tau \approx 1.618$, degenerate tree

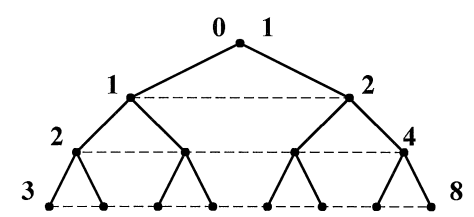

(a)

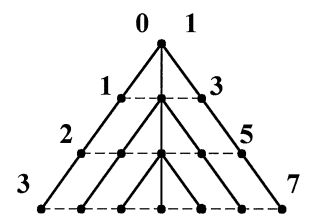

(c)

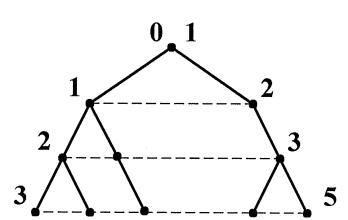

(b)

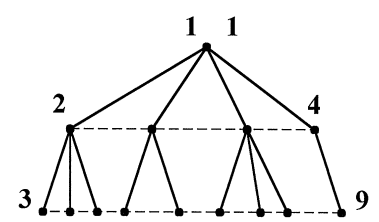

(d)

Fig. 3. Different types of hierarchical trees (the level number is indicated at left, corresponding number of nodes - at right): (a) regular tree with $j=2$; (b) Fibbonachi tree; (c) degenerate tree with $j=3$; and (d) irregular tree. 
with the only branching node per level and the tree of our primary concern - irregular tree. Let $k$ be the numbering index for the levels, so that $k$ increases from the top level to the bottom one. The variable

$$
s=s_{0}-k
$$

then defines the distance in the ultrametric space $[14,16]$. Geometrically, objects of this space correspond to the nodes of the bottom level $\left(k=s_{0}\right)$ of a Cayley tree. Since the distance between the nodes is defined by the number of steps to a common ancestor, the distance is eventually the level number (4.1), counted from the bottom.

As it can be seen in Fig. 3a, in the simplest case of regular tree with integer branching ratio $j$ the number of avalanches $N_{k}=j^{k}$ exponentially decays to zero with the distance $s$ between them:

$$
N(s)=N \exp (-s \ln j), \quad N \equiv j^{s_{0}} .
$$

In Eq. (4.2), equality (4.1) is used and the avalanche number $N$ is related to the total number of levels $s_{0}$. For the Fibbonachi tree (see Fig. 3b), where $N_{k}=v \tau^{k}, v \approx$ $1.171, \tau \approx 1.618$, we have

$$
N(s)=N \exp (-s \ln \tau), \quad N \equiv v \tau^{s_{0}} .
$$

When Eq. (4.3) is compared with Eq. (4.2), it is clear that the exponential decay remains unaltered in the case of fractional branching ratio and characterizes the regularity of the tree.

For the degenerate tree (see Fig. 3c) $N_{k}=(j-1) k+1$ and Eq. (4.1) provides the following linear dependence

$$
N(s)=N-(j-1) s, \quad N \equiv(j-1) s_{0}+1 .
$$

It can be shown that in the case of irregular tree, displayed in Fig. 3d, the power law dependence is realized:

$$
N_{k}=k^{a}, \quad a>1 .
$$

The latter can be regarded as an intermediate case between the exponential equations (4.2), (4.3) and linear equation (4.4) obtained for the limiting cases of regular and degenerate trees, respectively. Formally, approximation (4.5) means that a function $N(x)$ defined on the self-similar set of hierarchically subordinated avalanches is homogeneous, $N(k x)=k^{a} N(x)$. It is convenient to rewrite Eq. (4.5) in terms of the distance:

$$
N_{k}=N\left(1-s / s_{0}\right)^{a}, \quad N \equiv s_{0}^{a}, \quad a>1 .
$$

At the given value of crystallization thermal effect, $Q_{k}$, the heat current density between different levels $k$ is expressed by the generalized Onsager equality

$$
j_{k}=-\chi\left(Q_{k}\right) \frac{\mathrm{d} Q_{k}}{\mathrm{~d} k} .
$$

Here, within the multiplicative noise representation [17], the effective thermometric conductivity coefficient

$$
\chi(Q)=\chi Q^{\beta}
$$


is defined by the constant $\chi>0$ and the exponent $\beta$. The cornerstone of our approach is that total heat current at given level is independent of the hierarchical level

$$
j_{k} N_{k}=\text { const } \equiv J \text {. }
$$

Substitution of Eqs. (4.6)-(4.8) into Eq. (4.9) gives the expression for the crystallization heat effect

$$
Q_{k}=Q k^{-b}, \quad b=(a-1) /(1+\beta)>0
$$

normalized by the maximum value $Q \equiv Q_{k=1}$. Inserting Eq. (4.1), we get the dependence

$$
Q(s)=q\left(1-s / s_{0}\right)^{-b},
$$

where the heat effect at the bottom level $s=0$ is

$$
q \equiv Q s_{0}^{-b}=Q N^{-b / a} .
$$

In the general case, the condition of current conservation (4.9) is not satisfied and by taking into account Eqs. (4.10) and (4.12) we assume the scaling relation

$$
Q_{k}=N^{b / a} k^{-b} q_{k},
$$

where $q_{k}$ is a slowly varying function to be determined. According to Eqs. (4.7)-(4.9) it obeys the Landau-Khalatnikov equation

$$
\frac{\mathrm{d} x}{\mathrm{~d} \kappa}=-\frac{\partial V}{\partial x}
$$

where one denotes

$$
\kappa \equiv \ln k^{b}, \quad x \equiv q_{k} / q_{c}, \quad q_{c}^{1+\beta} \equiv(J / b \chi) N^{-(a-1) / a}
$$

and the effective potential is

$$
V=\frac{x^{1-\beta}}{1-\beta}-\frac{x^{2}}{2} .
$$

As indicated in Fig. 4, this potential reaches its maximum value $V_{0}=(1+\beta) / 2(1-\beta)$ at $x=1$ and decreases indefinitely at $x>1$. So, in order to initiate the process of explosive crystallization, a low intensity avalanche with $q<q_{c}$ at the bottom level needs to penetrate the barrier $V_{0}$. For a study of this process we proceed with stochastic Langevin equation with a white noise (cf. Eq. (4.14))

$$
\begin{aligned}
& \frac{\mathrm{d} x}{\mathrm{~d} \kappa}=-\frac{\partial V}{\partial x}+\zeta, \\
& \langle\zeta\rangle=0, \quad\left\langle\zeta(\kappa) \zeta\left(\kappa^{\prime}\right)\right\rangle=2 \chi \delta\left(\kappa-\kappa^{\prime}\right),
\end{aligned}
$$

where the noise intensity $\chi$ is reduced to the thermometric conductivity in Eq. (4.9).

The solutions of this equation are distributed in the ultrametric space according to the function $w(\kappa, x)$ that obeys the Fokker-Planck equation [18]

$$
\frac{\partial w}{\partial \kappa}+\frac{\partial j}{\partial x}=0, \quad j \equiv-w \frac{\partial V}{\partial x}-\chi \frac{\partial w}{\partial x} \text {. }
$$




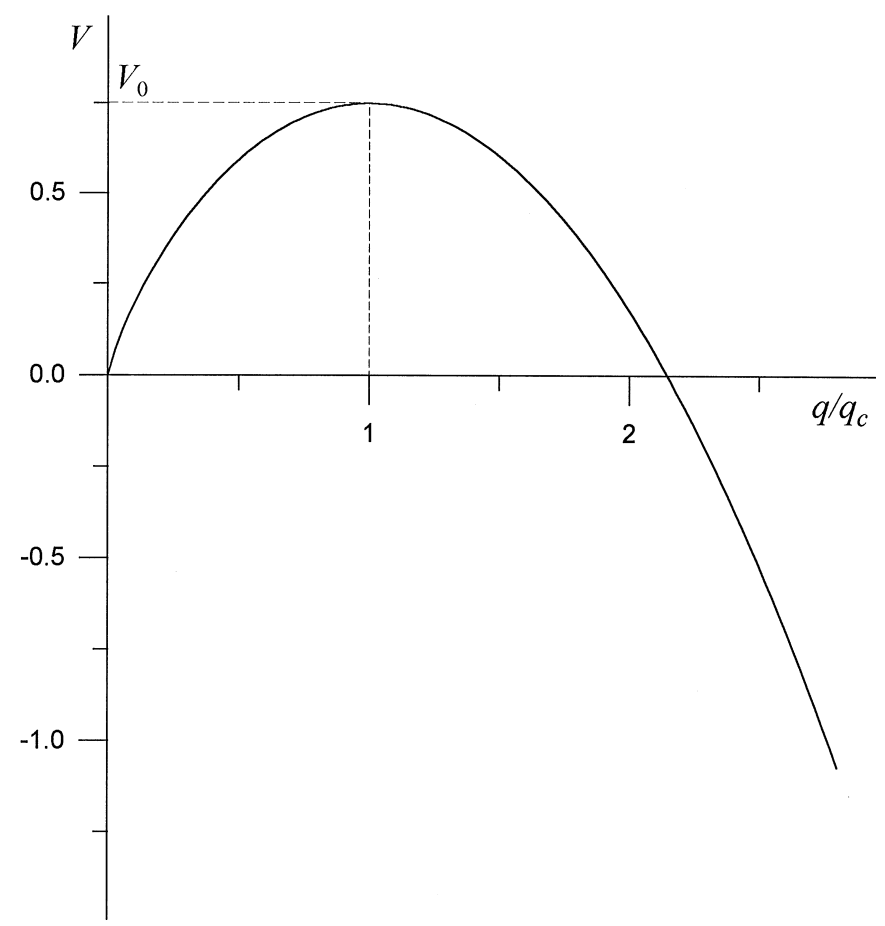

Fig. 4. The form of the effective potential (4.16) at $\beta=0.2$.

Since there is no current at the equilibrium state $(j=0)$, the distribution function

$$
w_{0}(x) \propto \exp (-V(x) / \chi)
$$

is dictated by potential (4.16). In the case of non-equilibrium steady state the probability density $w$ does not depend on the hierarchical level variable $\kappa$ and the current $j$ being constant, in compliance with conservation law (4.9), can take a non-zero value. In accordance with Eq. (4.19) the stationary $w(x)$ and the equilibrium $w_{0}(x)$ distributions are connected by the equation [19]

$$
\frac{w(q)}{w_{0}(q)}=\frac{j}{\chi} \int_{q / q_{c}}^{\infty} \frac{\mathrm{d} x}{w_{0}(x)},
$$

where the boundary condition $w \rightarrow 0$ at $q \rightarrow \infty$ is taken into account.

Given the heat effect $q$, Eq. (4.21) allows the current $j$ to be found. In trying to do it, special attention should be given to the fact that the heat $q$ is bounded from below, $q>G$ [12]. The appearance of the gap $G$ is the feature inherent in hierarchical ensemble of crystallization centers. Indeed, after merging of them within a hierarchical cluster of the size $s_{g}$, all $s$, such that $s<s_{g}$, as well as low heat effects with $q<q\left(s_{g}\right) \equiv$ $G$ appear to be dropped out of consideration (see Fig. 3). The expression for the 
current $j$ then can be derived from Eq. (4.21) with the second boundary condition $w(G)=w_{0}(G)$. The result reads

$$
j=2 \chi W\left[1+\operatorname{erf}\left(\sqrt{\frac{1+\beta}{2 \chi}}\left(1-\frac{G}{q_{c}}\right)\right)\right]^{-1},
$$

where the factor

$$
W \propto \exp \left(-V_{0} / \chi\right), \quad V_{0} \equiv \frac{1}{2} \frac{1+\beta}{1-\beta}
$$

gives the probability that the fluctuation will surmount the barrier $V_{0}$ of the potential (4.16). Eq. (4.22) shows that in the case of small gap, $G \ll q_{c}$, the current $j$ has the value $W \chi$ and it is doubled under $G=q_{c}$. It can be understood if we picture the effect of the gap as a mirror that reflects diffusing particles at the point $q=G$ : if $G \ll q_{c}$ a particle penetrating the barrier can move along both directions, but in the case of $G=q_{c}$ the mirror is placed at the point corresponding to the top of the barrier and all particles go down the side where the quantity $q$ grows indefinitely.

Given the current $j$ the stationary distribution function $w(x)$ is defined by Eq. (4.21), according to which, $w(x) \approx w_{0}(x)$ in the subcritical region $q<q_{c}$, while in the supercritical range $q \gg q_{c}$ we have $w_{0}(x) \gg w(x)$ due to indefinite increase of $w_{0}(x)$. As far as the stationary distribution is concerned, it can be derived from the current definition (4.19), where the last diffusion term is negligible for supercritical heats: $j \approx$ $-(\partial V / \partial x) w$. The result is that the probability $w(q)$ remains almost unaltered, $w(q) \approx$ $w\left(q_{c}\right)$, in the range from $q_{c}$ up to a boundary value $q_{g}$ and $w(q) \approx 0$ at $q>q_{g}$ [20]. The growth of $q_{g}$ is governed by the equation

$$
\frac{\mathrm{d} q_{g}}{\mathrm{~d} \kappa}=\chi \frac{q_{g}-q_{c}}{q_{g}^{2}}
$$

Since the above picture is essentially statistical, it enables the critical heat effect $q_{c}$ for the transition point to be found. Indeed, when the definition of the macroscopic current $J$ in Eq. (4.9) is compared to that of the microscopic current $j$ in Eq. (4.19), it is apparent that they differ from one another only by the factor $N^{(a-1) / a} \equiv s_{0}^{a-1}$ depending on the total number of embryos $N$ (see Eq. (4.6)). On this basis, the last expression of Eqs. (4.15) and (4.22) at $G=0, \chi \ll 1$ give the desired result

$$
q_{c}=Q \exp \left(-\frac{(1-\beta)^{-1}}{2 \chi}\right)
$$

where the pre-exponent factor $Q$ determines the probability of the barrier penetrating and cannot be calculated within the framework of the presented approach. Eq. (4.25) predicts the slow growth of the critical thermal effect $q_{c}$ of embryo with the thermometric conductivity coefficient $\chi$. 


\section{Time dependence of crystal formation probability}

Since the ensemble of hierarchically subordinated crystallization centers represents a self-similar set, the probability distribution of embryos $P(Q, s)$ in the course of SOC process is a homogeneous function of $s$ [12]

$$
P(Q, s)=s^{-\tau} w(q),
$$

where $w(q)$ is the stationary distribution of embryos and $\tau$ is the positive exponent. Physically, Eq. (5.1) implies that the heat effect $Q$, being measured by the scale $N^{b / a}$, equals the heat effect of an embryo formation $q$ in accordance with Eq. (4.12).

In this section, we aim to define the probability of hierarchical crystallization leading to the formation of a fractal cluster (see Fig. 1). As has been clarified in Section 4, this process can be conceived of as diffusion in ultrametric space that makes distribution (5.1) mounted. In order to find the conditional probability $\bar{P}(t)$ that no crystallization will begin at time $t$, one has to integrate over $s$ the distribution (5.1) weighted with the function

$$
p_{s}(t)=\exp (-t / t(s)), \quad t(s)=t_{0} \exp [Q(s) / \chi]
$$

descriptive of Debye relaxation with the time $t(s)$ governed by the barrier height $Q(s)$ (see Eq. (4.11)) and a microscopic time $t_{0}$ determined below. By using the steepest descent method, it is not difficult to derive the late time $\left(t \gg t_{e f}\right)$ asymptotic formula

$$
\overline{\mathscr{P}}(t)=\left(\frac{q}{\chi}\right)^{\tau / b}\left[1-\left(\frac{\chi}{q} \ln \frac{t}{t_{e f}}\right)^{-1 / b}\right]^{-\tau}, \quad t_{e f} \equiv \frac{\tau}{b}\left(\frac{q}{\chi}\right)^{1 / b} t_{0} .
$$

Eq. (5.3) has been obtained by assuming that the condition $1 \ll s_{m} \leqslant s_{0}$ is met, where $s_{m}$ denotes the location of the maximum of integrand and obeys the equation

$$
\frac{\chi \tau}{b q} \frac{(1-x)^{1+b}}{x}=\frac{t}{t_{0}} \exp \left(-\frac{q}{\chi}(1-x)^{-b}\right), \quad x \equiv \frac{s_{m}}{s_{0}} .
$$

Taking into consideration the scaling relation for the number of hierarchical levels $s_{0}$, which is the cut-off parameter [12]

$$
s_{0} \propto\left(q_{c}-q\right)^{-1 / \sigma}, \quad \sigma>0,
$$

we readily come to the conclusion that the condition is satisfied provided

$$
q-q_{c} \ll q, \quad t \gg t_{e f} \exp \left(\left(q_{c} / \chi\right)^{-1 / b}-1\right)^{-b} .
$$

Clearly, from Eq. (5.6) the heat effect $q$ in Eqs. (5.3) and (5.4) can be replaced by $q_{c}$. Note that in accordance with Eq. (5.3) the crystallization probability $\mathscr{P}(t) \equiv 1-\overline{\mathscr{P}}(t)$ logarithmically increases in time up to the value $\mathscr{P}=1-\left(q_{c} / \chi\right)^{\tau / b}$. The condition $\mathscr{P} \geqslant 0$ is satisfied if in Eq. (4.25) factor $Q=(\mathrm{e} / 2)(1-\beta)^{-1}$ and the thermometric conductivity coefficient are restricted by the maximum value $\chi_{0}=(1 / 2)(1-\beta)^{-1}$. 


\section{Discussion}

In accordance with the above approach, given in Section 3, the transition from the cold crystallization mode of amorphous material to the explosive crystallization mechanism represents the self-organization process realized as a first-order transition. The crystallization front velocity $u$ represents the order parameter, the temperatures difference $T$ at the crystallization front and thermostat acts as the conjugate field, and the difference $f$ of specific thermodynamic potentials of amorphous and crystalline states is the control parameter. Eqs. (3.9)-(3.11) are derived assuming the degrees of freedom $u, T$ and $f$ to be dissipative. In addition, the positive feedback between $u$ and $f$ is taken into consideration as the reason behind the self-organization, whereas the negative feedback between $u$ and $T$ is a manifestation of the Le Chatelier principle. The system is driven by the parameter $\theta$, whose value represents the thermal energy of crystallization (or externally inputted energy). When $\theta$ is above the critical value (3.16), the effective potential (3.15) assumes additional minimum value $-q<0$ at point $u=u_{e}$ and the maximum one $U$ at $u=u^{m}$ (stationary values of crystallization front velocity $u$ and thermodynamic factor $f$ are given by Eqs. (3.18) and (3.19)). The explosive crystallization process is preferable in potential provided the minimum effective potential becomes negative $(q>0)$. The height of the energy barrier $U$ of the effective potential (3.15) defines the characteristic time in the last Eq. (5.2) for hot crystallization center to be formed

$$
t_{0} \approx \tau_{D} \exp (U / \Delta)
$$

where $\Delta$ is the variance of $\theta$ and $\tau_{D} \sim 10^{-12} \mathrm{~s}$ is the Debye time.

The nucleating crystals form statistical ensemble of hierarchically subordinated objects, characterized by heat $q$ and distances $s$ in ultrametric space (crystalline cluster size [12]). Since the crystallization represents the effective diffusion over hierarchical tree nodes, similar to Brownian particle with coordinate $q$ at time $s$, the ensemble can be described by the Langevin equation (4.17) subjected to the noise equation (4.18) with $\chi$ being the effective diffusion coefficient (thermometric conductivity) and to the corresponding Fokker-Planck equation (4.19). The stationary heat distribution and the steady-state current are given by Eqs. (4.21) and (4.22). The condition of current conservation Eq. (4.9) yields the heat distribution (4.11) in the ultrametric space. The ensemble of embryos, being weakly dependent on $s$, is governed by the effective potential (4.16) that reaches its maximum at the critical heat (4.25) (see Fig. 4). So, the explosive crystallization requires supercritical heat effect, $q>q_{c}$, to surmount the barrier $V_{0}$ with the characteristic time (cf. Eq. (4.23))

$$
T \approx t_{0} \exp \left(V_{0} / \chi\right)
$$

This picture bears some resemblance to the formation process of supercritical embryo in the theory of a first-order phase transitions [19]. In the course of phase transformation the next stage is the growth of the embryo due to the diffusion increase of the heat 


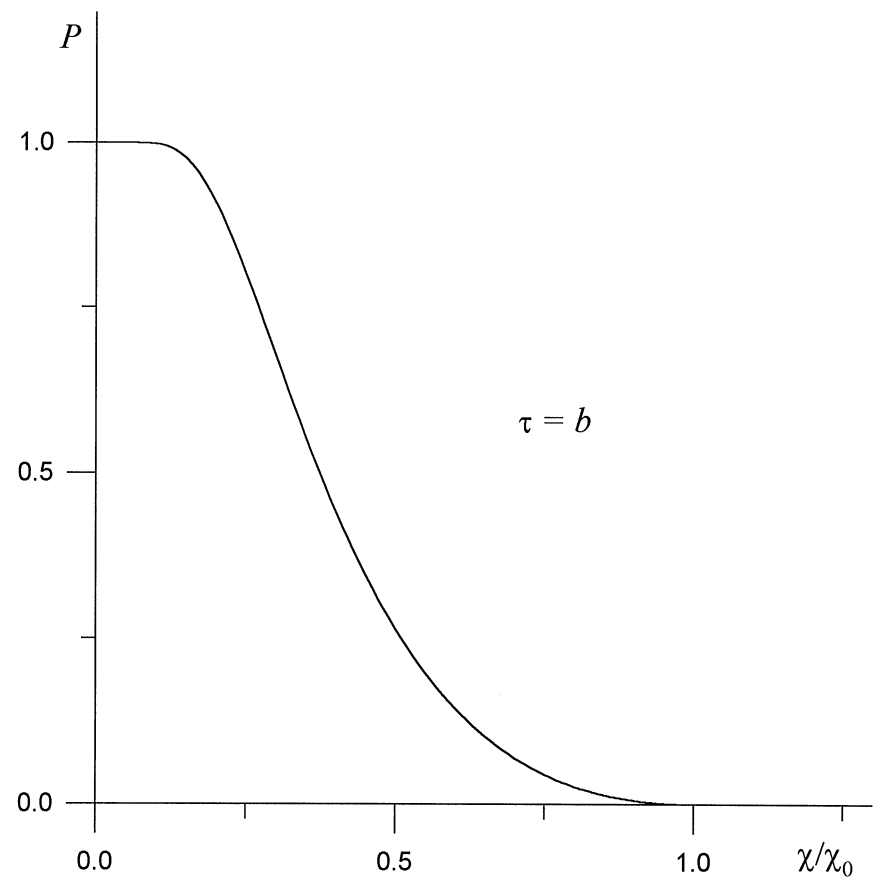

Fig. 5. The dependence of the maximum probability $\mathscr{P}$ of crystallization on the thermometric conductivity $\chi$.

effect $Q(s)$ in the ultrametric space. As a result, we have the logarithmically slow large time asymptotic for the probability of the total cluster formation

$$
\mathscr{P}(t)=1-\overline{\mathscr{P}}\left[1-\overline{\mathscr{P}}^{1 / \tau}\left(\ln \frac{t-T}{t_{e f}}\right)^{-1 / b}\right]^{-\tau}, \quad t_{e f} \equiv(\tau / b) \overline{\mathscr{P}}^{1 / \tau} t_{0},
$$

where time $t$ is counted from the instant $T$ (Eq. (6.2)), and $\overline{\mathscr{P}}$ is the minimum probability that no crystallization will occur

$$
\overline{\mathscr{P}}=\left(\frac{\chi_{0}}{\chi}\right)^{\tau / b} \exp \left[-\frac{\tau}{b}\left(\frac{\chi_{0}}{\chi}-1\right)\right] .
$$

From Eq. (6.4) the probability is determined by the ratio of the noise intensity $\chi$ (see Eq. (4.18)) to its maximum value $\chi_{0}=(1 / 2)(1-\beta)^{-1}$. The key point is that the maximum probability $\mathscr{P} \equiv 1-\overline{\mathscr{P}}$ of crystallization is completely suppressed under great thermometric conductivity coefficient $\chi$ (Fig. 5).

\section{References}

[1] V.P. Skripov, V.P. Koverda, Spontannaia kristallizatsiia pereokhlazhdennykh zhydkostei (Spontaneous Crystallization of Supercooled Liquids), Nauka, Moscow, 1984 (in Russian).

[2] L.N. Alexandrov, Kinetika kristallizatsii i perekristallizatsii poluprovodnikovykh pl'enok (Kinetics of Crystallization and Recrystallization of Semiconductors Films), Nauka, Novosibirsk, 1985 (in Russian). 
[3] V.P. Koverda, V.P. Skripov, Rasplavy 1 (4) (1987) 3 (in Russian).

[4] N.M. Bogdanov, V.P. Koverda, V.N. Skokov, V.P. Skripov, A.A. Dik, Kristallographia 33 (5) (1988) 1251 (in Russian).

[5] D.A. Kurtze, Phys. Rev. B 34 (1986) 1770.

[6] V.A. Shklovskii, V.M. Kuz'menko, Usp. Phys. Nauk 157 (2) (1989) 311 (in Russian).

[7] V.P. Koverda, Zh. Tekhn. Fiz. 64 (3) (1994) 62 (in Russian).

[8] J. Feder, Fractals, Plenum Press, New York, 1989.

[9] H. Haken, Synergetics, Springer, Berlin, 1983.

[10] A.I. Olemskoi, A.V. Khomenko, JETP 83 (1996) 1180.

[11] P. Bak, How Nature Works: the Science of Self-organized Criticality, University Press, Oxford, 1997.

[12] M. Parzuski, S. Maslov, P. Bak, Phys. Rev. E 53 (1996) 414.

[13] P. Bak, S. Boettcher, Physica D 107 (1997) 143.

[14] R. Rammal, G. Thoulouse, M.A. Virasoro, Rev. Mod. Phys. 58 (1986) 765.

[15] A.I. Olemskoi, A.D. Kiselev, Phys. Lett. A 247 (1998) 221; physics/9802035.

[16] A.I. Olemskoi, in: I.M. Khalatnikov (Ed.), Physics Reviews, vol. 18, Part 1, 1996, p. 1.

[17] A.I. Olemskoi, Phys. Usp. 41 (1998) 269.

[18] H. Risken, The Fokker-Planck Equation, Springer, Berlin, 1989.

[19] E.M. Lifshits, L.P. Pitaevskii, Phizicheskaia kinetika (Physical Kinetics), Nauka, Moscow, 1979 (in Russian).

[20] Yu.V. Mikhailova, L.A.Maksimov, ZhEksp. Teor. Fiz. 59 (1970) 1368 (in Russian). 